\title{
Prognostic value of preoperative inflammatory markers in patients with hepatocellular carcinoma underwent curative resection
}

\section{Wenlong Wu}

Fourth Military Medical University: Air Force Medical University

\section{Quancheng Wang}

Fourth Military Medical University Department of Social Sciences: Air Force Medical University

\section{Dandan Han}

Fourth Military Medical University: Air Force Medical University

\section{Jianhui Li}

Fourth Military Medical University: Air Force Medical University

\section{Ye Nie}

Fourth Military Medical University: Air Force Medical University

\section{Dongnan Guo}

: Xi'an Jiaotong University School of Pharmacy

\section{Long Yang}

Fourth Military Medical University: Air Force Medical University

\section{Kaishan Tao}

Fourth Military Medical University: Air Force Medical University

\section{Xuan Zhang}

Fourth Military Medical University: Air Force Medical University

Kefeng Dou ( $\nabla$ doukef@fmmu.edu.cn )

Fourth Military Medical University: Air Force Medical University https://orcid.org/0000-0003-3224-5072

\section{Research Article}

Keywords: Hepatocellular carcinoma, Platelet-to-lymphocyte ratio, Gamma-glutamyl transpeptidase-toplatelet ratio, Aminotransferase-to-lymphocyte ratio, Prognosis

Posted Date: April 26th, 2021

DOl: https://doi.org/10.21203/rs.3.rs-434954/v1

License: (c) (1) This work is licensed under a Creative Commons Attribution 4.0 International License. Read Full License 


\section{Abstract}

Background: The prognosis of hepatocellular carcinoma (HCC) is not optimistic. Our study focused on present inflammatory markers, including neutrophil-to-lymphocyte ratio (NLR), platelet-to-lymphocyte ratio (PLR), gamma-glutamyl transpeptidase-to-platelet ratio (GPR), aspartate aminotransferase-tolymphocyte ratio (ALR) and fibrinogen-to-albumin ratio (FAR), and aimed to explore their optimal combination for the prognosis of HCC after resection.

Methods: 347 HCC patients with curative resection were enrolled. The optimal cutoff values of the inflammatory markers were calculated using receiver operating characteristic (ROC) curve analysis, and used to divide patients into two groups whose differences were compared by Kaplan-Meier analysis. Cox univariate and multivariate analysis were used to analyze the independent prognostic inflammatory markers. $\mathrm{c} 2$ test was chosen to determine the relationship between independent prognostic inflammatory markers and clinicopathological features. We created the combined scoring models and evaluated them by Cox univariate and multivariate methods. The concordance index (C-index), Akaike information criterion (AIC) and likelihood ratio were calculated to compare the models. The selected optimal inflammatory markers and their combinations were tested in different stages of HCC by Kaplan-Meier analysis.

Results: ALR and GPR were independent prognostic factors for DFS; ALR, PLR, and GPR were independent prognostic factors for OS. The proposed GPR and ALR-GPR-PLR score models were independent predictors for DFS and OS, respectively.

Conclusion: The preoperative GPR and ALR-GPR-PLR score models were independent predictors for DFS and OS, respectively, and performed well in stratifying patients with HCC. The higher score in the model, the worse the prognosis was.

\section{Background}

Primary carcinoma of liver, known as one of the most commonly diagnosed cancers and the third leading cause of cancer death worldwide, includes intrahepatic cholangiocarcinoma and hepatocellular carcinoma (HCC) which made up for more than $50 \%$ of HCC patients cases in China $(1,2)$, has attracted more and more attention. Liver resection is still the mainstay of treatments for HCC patients. However, the clinical prognosis of HCC remains poor despite the advances in diagnostic and surgical techniques (3).

Within 5 years after curative resection, more than half of patients with HCC relapsed or migrated (4). The Barcelona Clinic Liver Cancer (BCLC) staging system, including recommendations for treatments, has been widely validated and is the most frequently used staging method $(5,6)$. The staging systems of American Joint Committee on Cancer (AJCC) and the China Liver Cancer (CNLC) are also commonly considered $(7,8)$. These staging systems have some limitations due to the heterogeneity of tumors. In addition, plasma alpha-fetoprotein (AFP) levels, a common tumor marker for HCC, remain within the 
normal range in $15-30 \%$ of advanced HCC patients(9). Therefore, more efficient prognostic indicators need to be explored to conduct active interventions to improve survival rates.

Inflammation promotes the tumorigenesis and the development of cancers(10). In China, the infection of Chronic hepatitis B virus (HBV) is the major pathogenic factor for HCC, whereas in western countries, the infection of hepatitis $\mathrm{C}$ virus $(\mathrm{HCV})$ accounts for the main risk factors(8). In recent years, some inflammatory indexes have been suggested to predict the prognosis of HCC. For example, the neutrophilto-lymphocyte ratio (NLR) and platelet-to-lymphocyte ratio (PLR) have been reported as the novel prognostic biomarkers for gastric cancer, colon cancer, cervical cancer, ovarian cancer and HCC (11-17). In addition, the gamma-glutamyl transpeptidase-to-platelet ratio (GPR) has been suggested to be related to the HCC prognosis $(18,19)$. Furthermore, other inflammatory markers, such as the aspartate aminotransferase-to-lymphocyte ratio (ALR) and fibrinogen-to-albumin ratio (FAR), have been suggested to predict the prognosis of $\mathrm{HCC}(20-22)$.

Although the above indexes have prognostic values, it is unclear that which marker has a better predictive role in HCC. Therefore, it will be of great significance to set up moderate models for the prognosis of HCC using these inflammatory markers.

Our study focused on comparing the effects of NLR, PLR, GPR, ALR and FAR in the prognosis of HCC patients with liver resection, in order to find the optimal combination and to establish models that can accurately predict prognosis.

\section{Methods}

From January 1st, 2014 to December 31st, 2017, 394 HCC patients with complete clinical materials and follow-up data who underwent hepatectomy were included. The followings are inclusion criteria: (1) pathological diagnosis of HCC; (2) surgical resection performed as the first treatment; (3) curative resection with a negative surgical margin; (4) at least 18 years old; and (5) complete preoperative laboratory examination data.

The following are exclusion criteria: (1) surgical treatment, local ablation, transarterial chemoembolization (TACE) or radiotherapy before hepatectomy; (2) other malignant tumors, AIDS, recent acute infection, or high fever; (3) recent administration of anti-inflammatory drugs or immunosuppressants; (4) rupture of HCC; and (5) incomplete clinical or pathological data. Finally, 347 HCC patients who underwent resection were included in this study. The flowchart of patient enrollment is shown in Fig. 1. Routine examination with the following was performed within one week before surgery: routine blood examination, blood biochemical series examination, chest X-ray, abdominal ultrasound, and computed tomography (CT) or magnetic resonance imaging (MRI). Clinical variables, including demographic data, medical history, complete blood count, albumin (Alb), AFP, aspartate aminotransferase (AST), gamma-glutamyl transpeptidase $(\mathrm{Y}-\mathrm{GT})$, total bilirubin (TB), prothrombin time (PT), fibrinogen, tumor pathological parameters, postoperative treatment, and Child-Turcotte-Pugh class (Child class), Eastern Cooperative Oncology Group (ECOG) performance status (PS), BCLC staging, AJCC staging, and 
CNLC staging, were collected. The equations of inflammatory markers NLR, PLR, GPR, ALR, and FAR were shown as follows:

NLR = neutrophil count/lymphocyte count ratio; PLR = platelet count/lymphocyte count ratio; GPR = $\gamma^{-}$ $\mathrm{GT} /$ platelet count ratio; $\mathrm{ALR}$ = aspartate aminotransferase/lymphocyte count ratio; FAR = fibrinogen/albumin ratio.

All patients were followed up through the electronic medical record system of the hospital and phone calls every three months starting from the date of surgery. Follow-up contents included recent health status, medications taken, hematologic tests, AFP levels, abdominal ultrasound, CT and MRI results. If abnormalities were found, we recommended the enhanced CT or MRI, accompany with further tests to confirm the patients' conditions. Recurrence was evaluated mainly according to the imaging. Disease-free survival (DFS) was defined by the time from the date of liver resection to the date of relapse or the date lost to follow-up. Overall survival (OS) was defined by the time from the date of liver resection to the date of HCC-associated death or the date lost to follow-up. The patients received our best medical care.

\section{Statistical Analysis}

SPSS for Windows version 22 (SPSS, Chicago, IL, USA) was used to analyze most of data in this study. Receiver operating characteristic (ROC) curves and the optimal cutoff values of the inflammatory markers were determined using MedCalc Statistical Software version 18.2.1 (MedCalc Software bvba, Ostend, Belgium). Continuous variables were presented as median ( $\mathrm{P}_{25}$ (lower quartile) - $\mathrm{P}_{75}$ (upper quartile)).

The optimal cutoff values were used to divide patients into two groups. The Kaplan-Meier method with log-rank test was chosen to measure the differences between two groups. The figures were drawn by GraphPad (GraphPad Software Inc. Prism Version 8.0.2, US). Cox univariate analysis was performed for inflammatory markers and other common clinical prognostic indicators. The variables of univariate analysis with $\mathrm{P}<0.1$ were imported into the multivariate analysis. Cox multivariate analysis was performed with the "Forward LR" method, and $\mathrm{P}<0.05$ (two-sides) indicated statistical significance. $\chi 2$ test was chosen to determine the relationship between independent prognostic inflammatory markers and clinicopathological features. Then, we created scoring models by combining the independent prognostic inflammatory markers. The combined scoring models integrating other common clinical prognostic indicators were analyzed by Cox univariate and multivariate analysis. To compare single inflammatory markers and the combined scoring models, R software (R Core Team (2020), Vienna, Austria) with the "survival" package (R package version 3.2-7, Terry M. Therneau, Patricia M. Grambsch (2000)) was used to calculate the concordance index (C-index) and likelihood ratio. The Akaike information criterion (AIC) was computed using the following formula: $A I C=2 k-2 \ln (L)$.

The prediction accuracy, goodness of fit and uniformity of the inflammatory markers and the combined scoring models were compared by the C-index, AIC and likelihood ratio. The selected optimal 
inflammatory markers and combined scoring models were tested in different stages of HCC by KaplanMeier analysis.

\section{Results}

\subsection{Demographics and tumor characteristics}

The present study included 347 patients, which $83.6 \%$ of patients (290 patients) were male and $16.4 \%$ were female (57 patients). Among the 347 patients, 300 patients' (86.5\%) hepatitis B surface antigen (HBsAg) were positive, 222 patients (64\%) had liver cirrhosis, 188 patients (54.2\%) received postoperative local ablation therapy or TACE, and 33 patients (9.5\%) used multiple kinase inhibitors (MKI). In terms of tumor parameters, 304 patients $(87.6 \%)$ had a tumor capsule; 305 patients $(87.9 \%)$ had a single tumor; 168 patients' $(48.4 \%)$ tumor size was $>5 \mathrm{~cm} ; 172$ patients $(49.6 \%)$ had microvascular invasion (MVI); and 29 (8.4\%), 283 (81.6\%), and 35 (10.1\%) patients had well, moderately and poorly differentiated tumor cells, respectively. To facilitate the analysis, 9 patients with BCLC stage 0 were merged into the BCLC stage A group, and 5 patients with AJCC stage IV were merged into the AJCC stage III group (Table 1). 
Table 1

Baseline characteristics of the 347 patients

\begin{tabular}{|c|c|c|c|c|c|}
\hline \multirow[t]{2}{*}{ Variable } & & Cases (\%) & Variable & & Cases (\%) \\
\hline & & \multicolumn{3}{|l|}{$\begin{array}{l}\text { /Median }\left(\mathrm{P}_{25^{-}}\right. \\
\left.\mathrm{P}_{75}\right)^{\mathrm{a}}\end{array}$} & 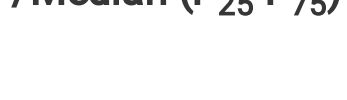 \\
\hline \multirow[t]{2}{*}{ Sex } & Male & $290(83.6)$ & Child class & $A$ & 335 (96.5) \\
\hline & Female & $57(16.4)$ & & $B$ & $12(3.5)$ \\
\hline \multirow[t]{2}{*}{ Age (years) } & $\geq 60$ & $95(27.4)$ & $\begin{array}{l}\text { Performance } \\
\text { status }\end{array}$ & 1 & $144(41.3)$ \\
\hline & $<60$ & $252(72.6)$ & & 0 & $203(58.2)$ \\
\hline \multirow[t]{2}{*}{$\mathrm{HBsAg}$} & Positive & $300(86.5)$ & BCLC staging & $A^{d}$ & $175(50.4)$ \\
\hline & Negative & $47(13.5)$ & & B & $17(4.9)$ \\
\hline \multirow[t]{2}{*}{ Liver cirrhosis } & Yes & $222(64)$ & & $\mathrm{C}$ & $155(44.7)$ \\
\hline & No & $125(36)$ & AJCC staging & प & $166(47.8)$ \\
\hline \multirow{2}{*}{$\begin{array}{l}\text { Portal vein } \\
\text { invasion }\end{array}$} & Yes & $21(6.1)$ & & ૫ & 136 (39.2) \\
\hline & No & 326 (93.9) & & $\square^{e}$ & $45(13)$ \\
\hline \multirow[t]{2}{*}{ Ascites } & Yes & $11(3.2)$ & CNLC staging & प & 291 (83.9) \\
\hline & No & $336(96.8)$ & & प & $33(9.5)$ \\
\hline \multirow[t]{2}{*}{ Ablation or TACE } & Yes & $188(54.2)$ & & प & $23(6.6)$ \\
\hline & No & $159(45.8)$ & Neutrophil count & & $3.6(2.56-4.72)$ \\
\hline \multirow[t]{2}{*}{ AFP (ng/ml) } & $>400$ & $132(38)$ & $\begin{array}{l}\text { Lymphocyte } \\
\text { count }\end{array}$ & & $1.45(1.03-1.84)$ \\
\hline & $\leq 400$ & $215(62)$ & Platelet count & & $150(109-205)$ \\
\hline \multirow[t]{2}{*}{ Tumor capsule } & Yes & $304(87.6)$ & $\mathrm{Y}-\mathrm{GT}$ & & $60(36-115)$ \\
\hline & No & $43(12.4)$ & AST & & $37(27-53)$ \\
\hline Tumor number & $\geq 2$ & $42(12.1)$ & Total bilirubin & & $15.2(12-21.2)$ \\
\hline
\end{tabular}

a. $\mathrm{P}_{25}-\mathrm{P}_{75}$ : (lower quartile - upper quartile) b. MVI: microvascular invasion c: MKI: multiple kinase inhibitor

d. Nine patients with BCLC stage 0 were merged into the BCLC stage A group

e. Five patients with AJCC stage IV were merged into the AJCC stage III group 


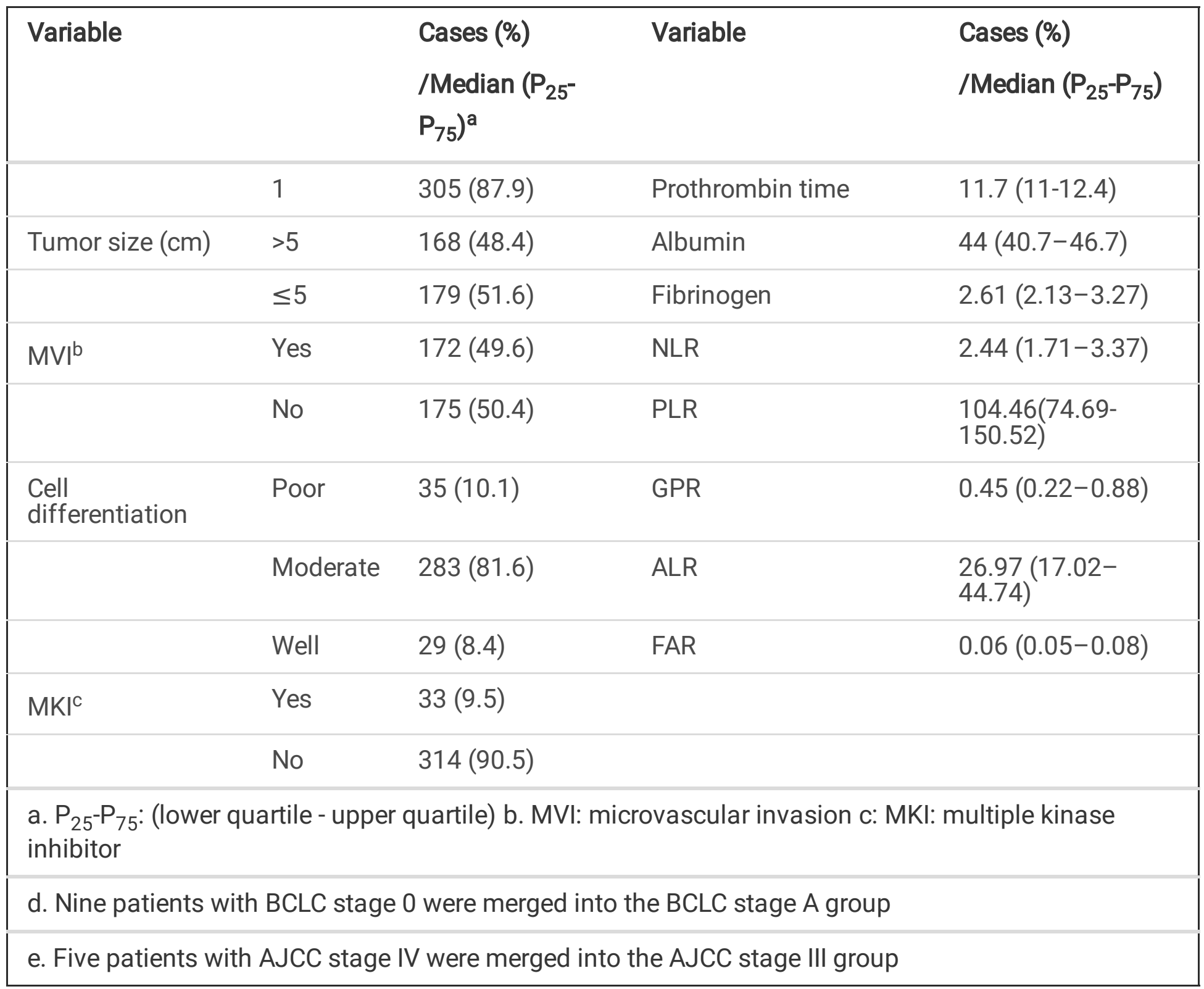

\subsection{Optimal cutoff values of the inflammatory markers}

The optimal cutoff values of NLR, PLR, GPR, ALR, and FAR were 2.33, 117.09, 0.48, 31, and 0.06, respectively. The areas under the curve (AUC) of those markers were $0.569,0.553,0.680,0.647$, and 0.632 (Fig. 2).

\subsection{OS and DFS rates}

The median follow-up time was 45 months. During the follow-ups, 216 patients (62.2\%) experienced recurrence, and 147 patients (42.4\%) passed away. The 1-, 3-, and 5-year DFS rates were 69.8\%, 41.5\%, and $30.8 \%$, and the $1-, 3$-, and 5 -year OS rates were $84.6 \%, 59.4 \%$, and $52.2 \%$, respectively. For the DFS rates, the $P$ values of different groups of NLR, PLR, GPR, ALR and FAR values were $0.088,0.082,<0.001$, $<0.001$ and $<0.001$, respectively. For the OS rate, the P values of different groups of NLR, PLR, GPR, ALR and FAR were $0.004,0.008,<0.001,<0.001$ and $<0.001$, respectively (Fig. 3).

\subsection{Independent prognostic factors for DFS and OS rates}


In the univariate analysis for DFS, variables with $\mathrm{P}<0.1$ including sex, age, portal vein invasion, ascites, AFP, tumor capsule, tumor size, tumor number, MVI, cell differentiation, MKI, NLR, PLR, GPR, ALR and FAR were selected for the multivariate analysis. According to the results of multivariate analysis, AFP $>400$ $\mathrm{ng} / \mathrm{ml}, \mathrm{ALR}>31, \mathrm{GPR}>0.48, \mathrm{MVl}$, absence of tumor capsule, and tumor size $>5 \mathrm{~cm}$ were independent prognostic factors for DFS (Table 2). 
Table 2

Univariate and multivariate analysis of DFS

\begin{tabular}{|c|c|c|c|c|c|}
\hline \multirow[t]{2}{*}{ Variable } & & \multicolumn{2}{|c|}{ Univariate analysis } & \multicolumn{2}{|c|}{ Multivariate analysis } \\
\hline & & HR (95\%Cl) & $\begin{array}{l}P \\
\text { value }\end{array}$ & $\mathrm{HR}(95 \% \mathrm{Cl})$ & $\begin{array}{l}\mathrm{P} \\
\text { value }\end{array}$ \\
\hline Sex & Male/Female & $\begin{array}{l}1.401(0.947- \\
2.072)\end{array}$ & 0.092 & & \\
\hline Age (years) & $\geq 60 /<60$ & $\begin{array}{l}0.767(0.560- \\
1.050)\end{array}$ & 0.097 & & \\
\hline HBsAg & Positive/Negative & $\begin{array}{l}1.317(0.874- \\
1.985)\end{array}$ & 0.189 & & \\
\hline Liver cirrhosis & Yes/No & $\begin{array}{l}1.080(0.819- \\
1.425)\end{array}$ & 0.586 & & \\
\hline $\begin{array}{l}\text { Portal vein } \\
\text { invasion }\end{array}$ & Yes/No & $\begin{array}{l}2.710(1.686- \\
4.357)\end{array}$ & $<.001$ & & \\
\hline Ascites & Yes/No & $\begin{array}{l}2.997(1.621- \\
5.539)\end{array}$ & $<.001$ & & \\
\hline Ablation or TACE & Yes/No & $\begin{array}{l}0.833(0.637- \\
1.089)\end{array}$ & 0.181 & & \\
\hline $\operatorname{AFP}(\mathrm{ng} / \mathrm{ml})$ & $>400 / \leq 400$ & $\begin{array}{l}1.674(1.279- \\
2.190)\end{array}$ & $<.001$ & $\begin{array}{l}1.377(1.042- \\
1.821)\end{array}$ & 0.025 \\
\hline Tumor capsule & No/Yes & $\begin{array}{l}2.146(1.497- \\
3.078)\end{array}$ & $<.001$ & $\begin{array}{l}1.864(1.289- \\
2.697)\end{array}$ & 0.001 \\
\hline Tumor number & $\geq 2 / 1$ & $\begin{array}{l}1.481(1.006- \\
2.181)\end{array}$ & 0.047 & & \\
\hline Tumor size (cm) & $>5 / \leq 5$ & $\begin{array}{l}1.766(1.348- \\
2.313)\end{array}$ & $\begin{array}{l}< \\
0.001\end{array}$ & $\begin{array}{l}1.440(1.090- \\
1.901)\end{array}$ & 0.010 \\
\hline$M V I^{\mathrm{a}}$ & Yes/No & $\begin{array}{l}1.649(1.259- \\
2.160)\end{array}$ & $<001$ & $\begin{array}{l}1.352(1.022- \\
1.789)\end{array}$ & 0.035 \\
\hline \multirow[t]{2}{*}{$\begin{array}{l}\text { Cell } \\
\text { differentiation }\end{array}$} & Moderate/Well & $\begin{array}{l}2.248(1.189- \\
4.251)\end{array}$ & 0.013 & & \\
\hline & Poor/Well & $\begin{array}{l}2.869(1.364- \\
6.031)\end{array}$ & 0.005 & & \\
\hline $\mathrm{MKI}^{\mathrm{b}}$ & No/Yes & $\begin{array}{l}0.596(0.395- \\
0.900)\end{array}$ & 0.014 & & \\
\hline Child class & $\mathrm{B} / \mathrm{A}$ & $\begin{array}{l}1.683(0.891- \\
3.177)\end{array}$ & 0.109 & & \\
\hline
\end{tabular}

a: MVI: microvascular invasion b: MKI: multiple kinase inhibitor 


\begin{tabular}{|c|c|c|c|c|c|}
\hline \multirow[t]{2}{*}{ Variable } & & \multicolumn{2}{|c|}{ Univariate analysis } & \multicolumn{2}{|c|}{ Multivariate analysis } \\
\hline & & $\mathrm{HR}(95 \% \mathrm{Cl})$ & $\begin{array}{l}P \\
\text { value }\end{array}$ & $\mathrm{HR}(95 \% \mathrm{Cl})$ & $\begin{array}{l}P \\
\text { value }\end{array}$ \\
\hline NLR & $>2.33 / \leq 2.33$ & $\begin{array}{l}1.264(0.965- \\
1.656)\end{array}$ & 0.089 & & \\
\hline PLR & $>117.09 / \leq 117.09$ & $\begin{array}{l}1.270(0.969- \\
1.664)\end{array}$ & 0.083 & & \\
\hline GPR & $>0.48 / \leq 0.48$ & $\begin{array}{l}2.327(1.770- \\
3.060)\end{array}$ & $<.001$ & $\begin{array}{l}1.931(1.445- \\
2.581)\end{array}$ & $<0.001$ \\
\hline ALR & $>31 / \leq 31$ & $\begin{array}{l}1.903(1.455- \\
2.488)\end{array}$ & $\begin{array}{l}<.001 \\
0.00\end{array}$ & $\begin{array}{l}1.438(1.083- \\
1.910)\end{array}$ & 0.012 \\
\hline FAR & $>0.06 / \leq 0.06$ & $\begin{array}{l}1.617(1.234- \\
2.117)\end{array}$ & $<.001$ & & \\
\hline
\end{tabular}

In the univariate analysis for OS, variables with $\mathrm{P}<0.1$ including HBV, portal vein invasion, ascites, AFP, tumor capsule, tumor size, tumor number, MVI, cell differentiation, NLR, PLR, GPR, ALR and FAR, were then entered into the Cox multivariate analysis. The results demonstrated that PLR $>117.09, A L R>31$, $\mathrm{GPR}>0.48, \mathrm{MVI}$, absence of tumor capsule, and tumor size $>5 \mathrm{~cm}$ were independent prognostic factors for OS (Table 3). 
Table 3

Univariate and multivariate analysis of OS

\begin{tabular}{|c|c|c|c|c|c|}
\hline \multirow[t]{2}{*}{ Variable } & & \multicolumn{2}{|c|}{ Univariate analysis } & \multicolumn{2}{|c|}{ Multivariate analysis } \\
\hline & & $\mathrm{HR}(95 \% \mathrm{Cl})$ & $\begin{array}{l}P \\
\text { value }\end{array}$ & $\mathrm{HR}(95 \% \mathrm{Cl})$ & $\begin{array}{l}P \\
\text { value }\end{array}$ \\
\hline Sex & Male/Female & $\begin{array}{l}1.478(0.912- \\
2.393)\end{array}$ & 0.113 & & \\
\hline Age (years) & $\geq 60 /<60$ & $\begin{array}{l}0.670(0.451- \\
0.996)\end{array}$ & 0.048 & & \\
\hline HBsAg & Positive/Negative & $\begin{array}{l}1.630(0.940- \\
2.736)\end{array}$ & 0.083 & & \\
\hline Liver cirrhosis & Yes/No & $\begin{array}{l}1.036(0.742- \\
1.448)\end{array}$ & 0.834 & & \\
\hline $\begin{array}{l}\text { Portal vein } \\
\text { invasion }\end{array}$ & Yes/No & $\begin{array}{l}3.757(2.256- \\
6.256)\end{array}$ & $\dot{0} 001$ & & \\
\hline Ascites & Yes/No & $\begin{array}{l}3.564(1.804- \\
7.039)\end{array}$ & $<.001$ & & \\
\hline Ablation or TACE & Yes/No & $\begin{array}{l}1.047(0.757- \\
1.449)\end{array}$ & 0.780 & & \\
\hline $\operatorname{AFP}(\mathrm{ng} / \mathrm{ml})$ & $>400 / \leq 400$ & $\begin{array}{l}1.844(1.334- \\
2.549)\end{array}$ & $\begin{array}{l}<.001 \\
0 .\end{array}$ & & \\
\hline Tumor capsule & No/Yes & $\begin{array}{l}2.710(1.811- \\
4.056)\end{array}$ & $<.001$ & $\begin{array}{l}2.119(1.394- \\
3.222)\end{array}$ & $\dot{0}_{0.001}$ \\
\hline Tumor number & $\geq 2 / 1$ & $\begin{array}{l}1.893(1.230- \\
2.915)\end{array}$ & 0.004 & & \\
\hline Tumor size (cm) & $>5 / \leq 5$ & $\begin{array}{l}2.170(1.553- \\
3.033)\end{array}$ & $<.001$ & $\begin{array}{l}1.646(1.157- \\
2.342)\end{array}$ & 0.006 \\
\hline$M V l^{a}$ & Yes/No & $\begin{array}{l}2.084(1.495- \\
2.906)\end{array}$ & $\begin{array}{l}<.001 \\
0\end{array}$ & $\begin{array}{l}1.678(1.195- \\
2.355)\end{array}$ & 0.003 \\
\hline \multirow[t]{2}{*}{$\begin{array}{l}\text { Cell } \\
\text { differentiation }\end{array}$} & Moderate/well & $\begin{array}{l}2.718(1.112- \\
6.647)\end{array}$ & 0.028 & & \\
\hline & Poor/well & $\begin{array}{l}3.703(1.375- \\
9.976)\end{array}$ & 0.010 & & \\
\hline$M K I^{b}$ & No/Yes & $\begin{array}{l}0.853(0.492- \\
1.480)\end{array}$ & 0.572 & & \\
\hline Child class & $\mathrm{B} / \mathrm{A}$ & $\begin{array}{l}1.380(0.609- \\
3.126)\end{array}$ & 0.440 & & \\
\hline
\end{tabular}

a: MVI: microvascular invasion b: MKI: multiple kinase inhibitor 


\begin{tabular}{|c|c|c|c|c|c|}
\hline \multirow[t]{2}{*}{ Variable } & & \multicolumn{2}{|c|}{ Univariate analysis } & \multicolumn{2}{|c|}{ Multivariate analysis } \\
\hline & & $\mathrm{HR}(95 \% \mathrm{Cl})$ & $\begin{array}{l}P \\
\text { value }\end{array}$ & $\mathrm{HR}(95 \% \mathrm{Cl})$ & $\begin{array}{l}P \\
\text { value }\end{array}$ \\
\hline NLR & $>2.37 / \leq 2.37$ & $\begin{array}{l}1.615(1.157- \\
2.253)\end{array}$ & 0.005 & & \\
\hline PLR & $\begin{array}{l}>117.09 / \\
\leq 117.09\end{array}$ & $\begin{array}{l}1.542(1.115- \\
2.133)\end{array}$ & 0.009 & $\begin{array}{l}1.465(1.024- \\
2.096)\end{array}$ & 0.037 \\
\hline GPR & $>0.48 / \leq 0.48$ & $\begin{array}{l}3.002(2.131- \\
4.230)\end{array}$ & $\begin{array}{l}<.001 \\
0 .\end{array}$ & $\begin{array}{l}2.554(1.757- \\
3.712)\end{array}$ & $\begin{array}{l}< \\
0.001\end{array}$ \\
\hline ALR & $>31 / \leq 31$ & $\begin{array}{l}2.255(1.628- \\
3.122)\end{array}$ & $\begin{array}{l}<.001 \\
0.01\end{array}$ & $\begin{array}{l}1.553(1.098- \\
2.197)\end{array}$ & 0.013 \\
\hline FAR & $>0.06 / \leq 0.06$ & $\begin{array}{l}1.984(1.425- \\
2.764)\end{array}$ & $<.001$ & & \\
\hline
\end{tabular}

\subsection{The relationship between independent prognostic inflammatory markers and clinicopathological features}

As ALR, GPR were independent prognostic factors for DFS; ALR, GPR, and PLR were independent prognostic factors for OS, the correlations of commonly clinicopathological variables with different groups of ALR, GPR and PLR were computed by the $\chi 2$ test, respectively. The results presented that ALR was correlated with portal vein invasion, ascites, AFP, tumor number, tumor size, MVI, BCLC, AJCC and CNLC staging; GPR was correlated with sex, portal vein invasion, ascites, AFP, tumor capsule, tumor number, tumor size, MVI, BCLC, AJCC and CNLC staging; PLR was correlated with sex, postoperative ablation or TACE, tumor capsule, tumor size, and BCLC staging [see Additional file 1].

\subsection{Creation and comparison of inflammatory scoring models for DFS and OS}

We generated models for the ALR, GPR and PLR score. At the same time, we created an ALR-GPR score model for DFS, and the ALR-GPR, ALR-PLR, GPR-PLR and ALR-GPR-PLR score (A-G-P score) models for OS. For simplicity of calculation, ALR $\leq 31, G P R \leq 0.48$ and PLR $\leq 117.09$ were defined as a score of 0 , and ALR $>31, G P R>0.48$ and PLR>117.09 were defined as a score of 1.

ALR, GPR and PLR score models consisted of scores of 0 and 1, models for the ALR-GPR, ALR-PLR and GPR-PLR score consisted of scores of $\leq 1$ and 2; the A-G-P score model consisted of scores of $\leq 1,2$ and 3 (Table 4). 
Table 4

Models of inflammatory markers

Model for

OS

\begin{tabular}{|c|c|c|c|c|c|}
\hline \multirow[t]{2}{*}{ ALR score } & $A L R>31$ & 1 & ALR score & $A L R>31$ & 1 \\
\hline & $A L R \leq 31$ & 0 & & $A L R \leq 31$ & 0 \\
\hline \multirow[t]{2}{*}{ GPR score } & GPR $>0.48$ & 1 & GPR score & GPR $>0.48$ & 1 \\
\hline & $\mathrm{GPR} \leq 0.48$ & 0 & & $\mathrm{GPR} \leq 0.48$ & 0 \\
\hline \multirow[t]{2}{*}{ PLR score } & PLR > 117.09 & 1 & $\begin{array}{l}\text { ALR-GPR } \\
\text { score }\end{array}$ & $\begin{array}{l}\mathrm{ALR}>31 \text { and } \mathrm{GPR}> \\
0.48\end{array}$ & 2 \\
\hline & PLR $\leq 117.09$ & 0 & & others & $\leq 1$ \\
\hline \multirow{2}{*}{$\begin{array}{l}\text { ALR-GPR } \\
\text { score }\end{array}$} & $\mathrm{ALR}>31$ and $\mathrm{GPR}>0.48$ & 2 & & & \\
\hline & others & $\leq 1$ & & & \\
\hline \multirow[t]{2}{*}{$\begin{array}{l}\text { ALR-PLR } \\
\text { score }\end{array}$} & $\begin{array}{l}\text { ALR }>31 \text { and PLR }> \\
117.09\end{array}$ & 2 & & & \\
\hline & others & $\leq 1$ & & & \\
\hline \multirow[t]{2}{*}{$\begin{array}{l}\text { GPR-PLR } \\
\text { score }\end{array}$} & $\begin{array}{l}\text { GPR }>0.48 \text { and } P L R> \\
117.09\end{array}$ & 2 & & & \\
\hline & others & $\leq 1$ & & & \\
\hline \multirow[t]{3}{*}{ A-G-P score ${ }^{a}$} & $\begin{array}{l}\text { ALR }>31, \text { GPR }>0.48 \\
\text { and } P L R>117.09\end{array}$ & 3 & & & \\
\hline & $\begin{array}{l}A L R>31, G P R>0.48, \\
\text { and } P L R \leq 117.09 ; \\
\text { or } A L R>31, P L R>117.09 \text {, } \\
\text { and } G P R \leq 0.48 ; \\
\text { or } P L R>117.09, \text { GPR > } \\
0.48, \\
\text { and } A L R \leq 31\end{array}$ & 2 & & & \\
\hline & others & $\leq 1$ & & & \\
\hline
\end{tabular}

a: A-G-P score: ALR-GPR-PLR score

We further verified whether the above combined scoring models (as categorical variables) w ere independent predictors of prognosis through univariate and multivariate analysis. Obviously, single inflammatory marker model ALR, GPR and PLR score were independent predictors. When verifying one
Score

Model for DFS 
combined scoring model, we no longer put the individual inflammatory markers that make up the model into analyses in order to exclude their interactions. The results demonstrated that all of the ALR-GPR, ALR-PLR, GPR-PLR and A-G-P score models were independent predictors for OS, and the ALR-GPR score was an independent predictive factor for DFS [see Additional file 2-6].

Then, we compared the single inflammatory marker models and the combined scoring models by the Cindex, AIC and likelihood ratio. For OS, compared with other models, the A-G-P score model had the smallest AIC value (1569.94), the largest C-index value $(0.653,95 \% \mathrm{Cl}: 0.610-0.696)$ and the largest likelihood ratio (50.48), suggesting that the A-G-P score model has a better prediction accuracy, goodness of fit, and uniformity in predicting the survival of patients who underwent resection. In terms of DFS, the single inflammatory marker model, the GPR score had the smallest AIC value (2264.32), the largest Cindex value $(0.605,95 \% \mathrm{Cl}$ : $0.572-0.638)$ and largest likelihood ratio (37.39), suggesting that the GPR score model has a better prediction (Table 5).

Table 5

Comparison of models for OS/DFS

\begin{tabular}{|llll|}
\hline Model & AIC & C-index & Likelihood ratio \\
\hline OS & & & \\
\hline ALR score & 1596.53 & 0.61 & 23.89 \\
\hline GPR score & 1578.07 & 0.64 & 42.34 \\
\hline PLR score & 1613.68 & 0.56 & 6.73 \\
\hline ALR-GPR score & 1574.60 & 0.61 & 30.96 \\
\hline ALR-PLR score & 1598.11 & 0.59 & 22.30 \\
\hline GPR-PLR score & 1585.71 & 0.60 & 34.70 \\
\hline A-G-P score & 1569.94 & 0.65 & 50.48 \\
\hline DFS & & & \\
\hline ALR score & 2280.10 & 0.59 & 21.61 \\
\hline GPR score & 2264.32 & 0.61 & 37.39 \\
\hline ALR-GPR score & 2272.73 & 0.59 & 28.98 \\
\hline a: A-G-P score: ALR-GPR-PLR score & \\
\hline
\end{tabular}

\subsection{The effect of stratification of the A-G-P score model and GPR score model on different stages of HCC}

The A-G-P score model has a good discriminating ability for OS in the whole population with a statistically significant difference (score $\leq 1$ /score $2, P<0.001$; score $\leq 1$ / score $3, P<0.001$; score $2 /$ score $3, P<0.001)$. For the pairwise comparison of the A-G-P score, we used Bonferroni correction, and $P<0.0167(a=0.05 / 3)$ was considered statistically significant between the different scores. Regarding 
BCLC staging, score $\leq 1 /$ score $3(P=0.007)$ in stage $A$; score $\leq 1$ /score $2(P=0.004)$, score $\leq 1 /$ score $3(P$ $<0.001)$, and score $2 /$ score $3(P<0.004)$ in stage $C$ were significantly different. Regarding AJCC staging, score $\leq 1 /$ score $3(P<0.001)$ and score $2 /$ score $3(P=0.006)$ in stage $\mathrm{I}$, score $\leq 1 /$ score $2(P<0.001)$ and score $\leq 1 /$ score $3(P<0.001)$ in stage II were significantly different. For CNLC staging, score $\leq 1 /$ score 2 $(P<0.001)$, score $\leq 1 /$ score $3(P<0.001)$, and score 2 /score $3(P<0.001)$ in stage $\nabla$ showed significant differences for the prognosis of OS (Fig. 4).

The GPR score model was a good differentiator for DFS in the whole population, with $P<0.001$. For different stages of HCC, in BCLC stage $C(P<0.001)$, AJCC stage II $(P<0.001)$, AJCC stage III $(P=0.004)$, CNLC stage I $(P<0.001)$, and CNLC stage III $(P=0.027)$, different scores of GPR score model showed significant differences for DFS (Fig. 5).

\section{Discussion}

Cancer-related chronic inflammation is a remarkable characteristic of cancer and promotes the metastasis. The persistent presence of inflammatory cells in the tumor establishes cross-talk with tumor cells that may lead to the conversion of phenotypes to tumor support cells(23). The long-term effect of $\mathrm{HBV}$ or HCV has a key impact on the development of HCC. At present, inflammatory scores have been suggested to have predictive values for the prognosis of HCC. NLR and GPR were verified as inflammation-related factors in predicting the survival of patients with HCC after liver resection (24).

The number of neutrophil independently correlated with TNM staging, performance status and poorer prognosis, indicating the key role of neutrophils in $\mathrm{HCC}(25)$. High values of preoperative PLR may be an adverse prognostic factor for OS and DFS in HCC patients. However, elevated PLR value was not highly associated with the vascular invasion, tumor numbers, AFP levels or poor tumor grades (17).

In addition, patients with a high value of ALR had unsatisfactory prognosis, and ALR was an independent prognostic factor for $\mathrm{HCC}(21)$. In our study, the elevated ALR was associated with portal vein invasion, AFP levels, tumor numbers, tumor sizes and MVI, and was an independent predictor for both DFS and OS. It was reported that elevated FAR was associated with poor prognosis and higher relapse rates in HCC patients(22); nevertheless, our study demonstrated that the FAR was not a prognostic factor in HCC.

Some studies revealed that NLR is a predictive factor in HCC, but it was excluded after multivariate analysis in our research. To eliminate the possible influence of other inflammatory markers on NLR in the Cox multivariate analysis, we further conducted a Cox multivariate analysis with only NLR as an inflammatory marker and other significant univariate variables. The result supported our previous findings that the NLR was not an independent prognostic factor for OS [see Additional file 7]. A retrospective study proposed that the inflammatory markers of PLR and CRP (but not NLR) have prognosis values, possibly because they reflected the value of parameters representative of tumor growth and aggressiveness(26). To some extent, these results are consistent with our research. 
In the present study, inflammatory markers were found to be effective and reliable indicators for the prognosis of $\mathrm{HCC}(27)$, but the effect of inflammatory markers on prognosis in $\mathrm{HCC}$ after resection is still confusing. In our research, we examined the independent predictors of HCC patients with liver resection.

ALR, GPR and PLR were independent prognostic factors for OS. Elevated serum AST values usually indicate extensive damage to the liver parenchyma and poor prognosis. The host's immune response to tumors depends on lymphocytes. Lymphopenia may lead to a poor result of lymphocyte-mediated tumor immune response, and a higher risk of cancer relapses (28). Therefore, a higher ALR value predicts a worse prognosis. As a component of GPR, the elevation of $\mathrm{Y}-\mathrm{GT}$ indicates poor liver function. Therefore, the higher $\mathrm{Y}-\mathrm{GT}$ is, the greater value of GPR, indicating poorer prognosis in HCC. In addition, another problem should be considered that GPR and PLR are both independent factors affecting the survival of HCC. In calculating the GPR, the platelet count is in the denominator, which means that the larger platelet count is, the smaller the value of GPR, indicating a better prognosis. However, in calculating the PLR, the platelet count is in the numerator, indicating that the larger the platelet count is, the worse the prognosis, which is contradictory to the results of GPR. Cancer cells can activate platelets, and the activation of platelets can lead to cancer-related inflammation, metastasis and cancer progression(29). In contrast, platelets play important roles in cancer progression at different stages. For example, platelets suppresses tumors via the downregulation of TC genes and induction of tumor cell apoptosis in early stages(30). Thus, platelets have the dual roles of promoting tumor development and inhibiting tumor growth by influencing the tumor microenvironment and their powerful secretory function.

DFS has independent prognostic factors, including ALR, GPR and AFP. Worth to be mentioned about, AFP is not considered as an independent factor for OS, suggesting that a high AFP level (AFP > $400 \mathrm{ng} / \mathrm{ml}$ ) has a greater effect on recurrence than on survival.

The A-G-P and GPR score models were selected as the optimal models for OS and DFS, respectively, by comparing the models using the AIC, C-index and likelihood ratio. These two models not only performed well in the total population but also showed good stratification ability for most of the different stages of HCC. In particular, in the A-G-P score model, 3 groups were significantly different in BCLC stage $C$ and

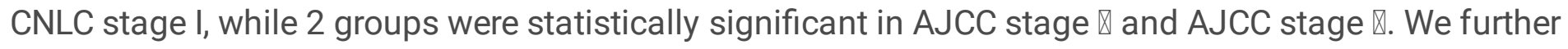
analyzed why this scoring system performed well in both BCLC stage $C$ (advanced stage) and CNLC stage I (early stage), and found that among the 155 patients with BCLC stage C, 132 patients were assigned to BCLC stage $C$ simply because of their PS of 1 point. The PS point value is easily affected by the patient's possible unclear expression and the subjectivity of the surgeon. CNLC stage I includes patients with PS values of 1 and 2 points, and 116 patients belonging to BCLC stage $C$ will be reassigned to CNLC stage I according to the CNLC criterion. Thus, the above question will become easy to understand. With respect to the GPR score model for DFS, the model shows good discrimination ability in

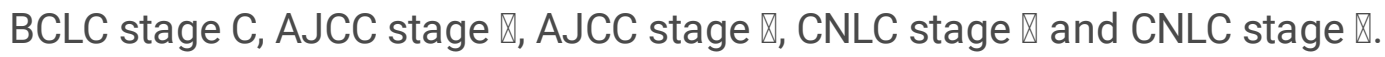

Our research still have room for improvements. Firstly, the research is a single-center retrospective study, there might be biases in sample selection. Next, the portal hypertension probably has impacts on 
hematological indicators, but due to the lack of direct data on portal hypertension measurements, we did not take portal hypertension into the consideration.

\section{Conclusions}

At present, the prognosis of HCC is mainly based on staging systems and prognostic indicators, however, it is not sufficient to judge the prognosis of HCC by these criteria alone, due to the prognosis of HCC is not only determined by tumor itself, but also influenced by the patients' liver function. Our study focused on critical indexes of liver function and representative inflammatory cells, and found that changes in these markers have a significant impact on the prognosis of HCC.

In summary, our study demonstrated for the first time that the ALR-GPR-PLR score model was an independent predictor for OS and performed well in stratifying patients with $\mathrm{HCC}$. We also proposed the GPR score model and confirmed its effect on DFS. These models are readily available and affordable, and could supplement and improve the existing prognosis criteria of HCC, providing guidance for the postoperative interventions of patients with HCC.

\section{List Of Abbreviations}

ALR Aspartate aminotransferase-to-lymphocyte ratio

A-G-P score model ALR-GPR-PLR score model

AIC Akaike information criterion

C-index Concordance index

DFS Disease-free survival

FAR Fibrinogen-to-albumin ratio

GPR Gamma-glutamyl transpeptidase-to-platelet ratio

HCC Hepatocellular carcinoma

NLR Neutrophil-to-lymphocyte ratio

OS Overall survival

PLR Platelet-to-lymphocyte ratio

ROC Receiver operating characteristic

\section{Declarations}




\section{Ethics approval and consent to participate}

Our research was approved by the Medical Ethics Committee of the First Affiliated Hospital of the Air Force Medical University (KY20202115-C-1).

\section{Consent for publication}

Not applicable

\section{Availability of data and materials}

All data generated or analysed during this study are included in this published article [and its supplementary information files].

\section{Competing interests}

The authors declare that they have no competing interests.

\section{Funding}

This study was supported by the National Key Research and Development program of China (2016YFC0905902, 2017YFC1103703, 2016YFA0102100) and the National Natural Science Foundation of China $(82070671,81870446,81970566,82070681,81900571)$.

\section{Authors' contributions}

Wenlong Wu, Quancheng Wang, and Dandan Han contributed equally to this work.

Kefeng Dou, Kaishan Tao, and Xuan Zhang designed the study.

Jianhui Li, Ye Nie, and Long Yang performed the analysis.

Dongnan Guo draw the figures and revised the article.

\section{Acknowledgements}

Special thanks to Dr. Yibin Guo (Department of Health Statistics, Second Military Medical University) for careful guidance in statistics.

\section{References}

1. Sung H, Ferlay J, Siegel RL, Laversanne M, Soerjomataram I, Jemal A, et al. Global cancer statistics 2020: GLOBOCAN estimates of incidence and mortality worldwide for 36 cancers in 185 countries. CA Cancer J Clin. 2021. 
2. Chen W, Zheng R, Baade P, Zhang S, Zeng H, Bray F, et al. Cancer statistics in China, 2015. Cancer J Clin. 2016;66(2):115-32.

3. Villanueva A, Hoshida Y, Battiston C, Tovar V, Sia D, Alsinet C, et al. Combining clinical, pathology, and gene expression data to predict recurrence of hepatocellular carcinoma. Gastroenterology. 2011;140(5):1501-12.e2.

4. Bruix J, Gores G, Mazzaferro V. Hepatocellular carcinoma: clinical frontiers and perspectives. Gut. 2014;63(5):844-55.

5. Bruix J, Reig M, Sherman M. Evidence-Based Diagnosis, Staging, and Treatment of Patients With Hepatocellular Carcinoma. Gastroenterology. 2016;150(4):835-53.

6. EASL Clinical Practice Guidelines. Management of hepatocellular carcinoma. Journal of hepatology. 2018;69(1):182-236.

7. Chun Y, Pawlik T, Vauthey J. 8th Edition of the AJCC Cancer Staging Manual: Pancreas and Hepatobiliary Cancers. Ann Surg Oncol. 2018;25(4):845-7.

8. Xie D, Ren Z, Zhou J, Fan J, Gao Q. 2019 Chinese clinical guidelines for the management of hepatocellular carcinoma: updates and insights. Hepatobiliary surgery and nutrition. 2020;9(4):452 63.

9. Han L, Lv Y, Guo H, Ruan Z, Nan K. Implications of biomarkers in human hepatocellular carcinoma pathogenesis and therapy. World journal of gastroenterology. 2014;20(30):10249-61.

10. Greten F, Grivennikov S. Inflammation and Cancer: Triggers, Mechanisms, and Consequences. Immunity. 2019;51(1):27-41.

11. Zhang Y, Lu J, Du Y, Feng C, Wang L, Chen M. Prognostic value of neutrophil-to-lymphocyte ratio and platelet-to-lymphocyte ratio in gastric cancer. Medicine. 2018;97(12):e0144.

12. Hu Z, Tan S, Chen S, Qin S, Chen H, Qin S, et al. Diagnostic value of hematological parameters platelet to lymphocyte ratio and hemoglobin to platelet ratio in patients with colon cancer. Clin Chim Acta. 2020;501:48-52.

13. Ma J, Ke L, Liu Q. The pretreatment platelet-to-lymphocyte ratio predicts clinical outcomes in patients with cervical cancer: A meta-analysis. Medicine. 2018;97(43):e12897.

14. Zhao Z, Zhao X, Lu J, Xue J, Liu P, Mao H. Prognostic roles of neutrophil to lymphocyte ratio and platelet to lymphocyte ratio in ovarian cancer: a meta-analysis of retrospective studies. Archives of gynecology obstetrics. 2018;297(4):849-57.

15. He C, Zhang Y, Cai Z, Lin X. The prognostic and predictive value of the combination of the neutrophilto-lymphocyte ratio and the platelet-to-lymphocyte ratio in patients with hepatocellular carcinoma who receive transarterial chemoembolization therapy. Cancer management research. 2019;11:1391400.

16. Uchinaka E, Amisaki M, Morimoto M, Tokuyasu N, Sakamoto T, Honjo S, et al. Utility and Limitation of Preoperative Neutrophil Lymphocyte Ratio as a Prognostic Factor in Hepatocellular Carcinoma. Yonago acta medica. 2018;61(4):197-203. 
17. Ma W, Zhang P, Qi J, Gu L, Zang M, Yao H, et al. Prognostic value of platelet to lymphocyte ratio in hepatocellular carcinoma: a meta-analysis. Scientific reports. 2016;6:35378.

18. Dai T, Deng M, Ye L, Liu R, Lin G, Chen X, et al. Prognostic value of combined preoperative gammaglutamyl transpeptidase to platelet ratio and fibrinogen in patients with HBV-related hepatocellular carcinoma after hepatectomy. American journal of translational research. 2020;12(6):2984-97.

19. Ke M, Zhang M, Su Q, Wei S, Zhang J, Wang Y, et al. Gamma-glutamyl transpeptidase to platelet ratio predicts short-term outcomes in hepatocellular carcinoma patients undergoing minor liver resection. J Surg Res. 2018;231:403-10.

20. Zhao L, Yang D, Ma X, Liu M, Wu D, Zhang X, et al. The Prognostic Value of aspartate aminotransferase to lymphocyte ratio and systemic immune-inflammation index for Overall Survival of Hepatocellular Carcinoma Patients Treated with palliative Treatments. J Cancer. 2019;10(10):2299-311.

21. Chen $Y$, He C, Wen T, Yan L, Yang J. The prognostic value of aspartate aminotransferase-tolymphocyte ratio index in early-stage hepatocellular carcinoma after hepatectomy: A propensityscore matched analysis. Asia-Pacific journal of clinical oncology. 2020.

22. Xu Q, Yan Y, Gu S, Mao K, Zhang J, Huang P, et al. A Novel Inflammation-Based Prognostic Score: The Fibrinogen/Albumin Ratio Predicts Prognoses of Patients after Curative Resection for Hepatocellular Carcinoma. Journal of immunology research. 2018;2018:4925498.

23. Suarez-Carmona M, Lesage J, Cataldo D, Gilles C. EMT and inflammation: inseparable actors of cancer progression. Molecular oncology. 2017;11(7):805-23.

24. Wang Y, Sun K, Shen J, Li B, Kuang M, Cao Q, et al. Novel Prognostic Nomograms Based on Inflammation-Related Markers for Patients with Hepatocellular Carcinoma Underwent Hepatectomy. Cancer research treatment: official journal of Korean Cancer Association. 2019;51(4):1464-78.

25. Margetts J, Ogle L, Chan S, Chan A, Chan K, Jamieson D, et al. Neutrophils: driving progression and poor prognosis in hepatocellular carcinoma? British journal of cancer. 2018;118(2):248-57.

26. Suner A, Carr B, Akkiz H, Uskudar O, Kuran S, Tokat Y, et al. Inflammatory markers C-reactive protein and PLR in relation to HCC characteristics. Journal of translational science. 2019;5(3).

27. Huang L, Mo Z, Hu Z, Zhang L, Qin S, Qin X, et al. Diagnostic value of fibrinogen to prealbumin ratio and gamma-glutamyl transpeptidase to platelet ratio in the progression of AFP-negative hepatocellular carcinoma. Cancer Cell Int. 2020;20:77.

28. Liao W, Zhang J, Zhu Q, Qin L, Yao W, Lei B, et al. Preoperative Neutrophil-to-Lymphocyte Ratio as a New Prognostic Marker in Hepatocellular Carcinoma after Curative Resection. Translational oncology. 2014;7(2):248-55.

29. Palacios-Acedo A, Mège D, Crescence L, Dignat-George F, Dubois C, Panicot-Dubois L, Platelets. Thrombo-Inflammation, and Cancer: Collaborating With the Enemy. Frontiers in immunology. 2019;10:1805.

30. Michael J, Wurtzel J, Mao G, Rao A, Kolpakov M, Sabri A, et al. Platelet microparticles infiltrating solid tumors transfer miRNAs that suppress tumor growth. Blood. 2017;130(5):567-80. 
Figures

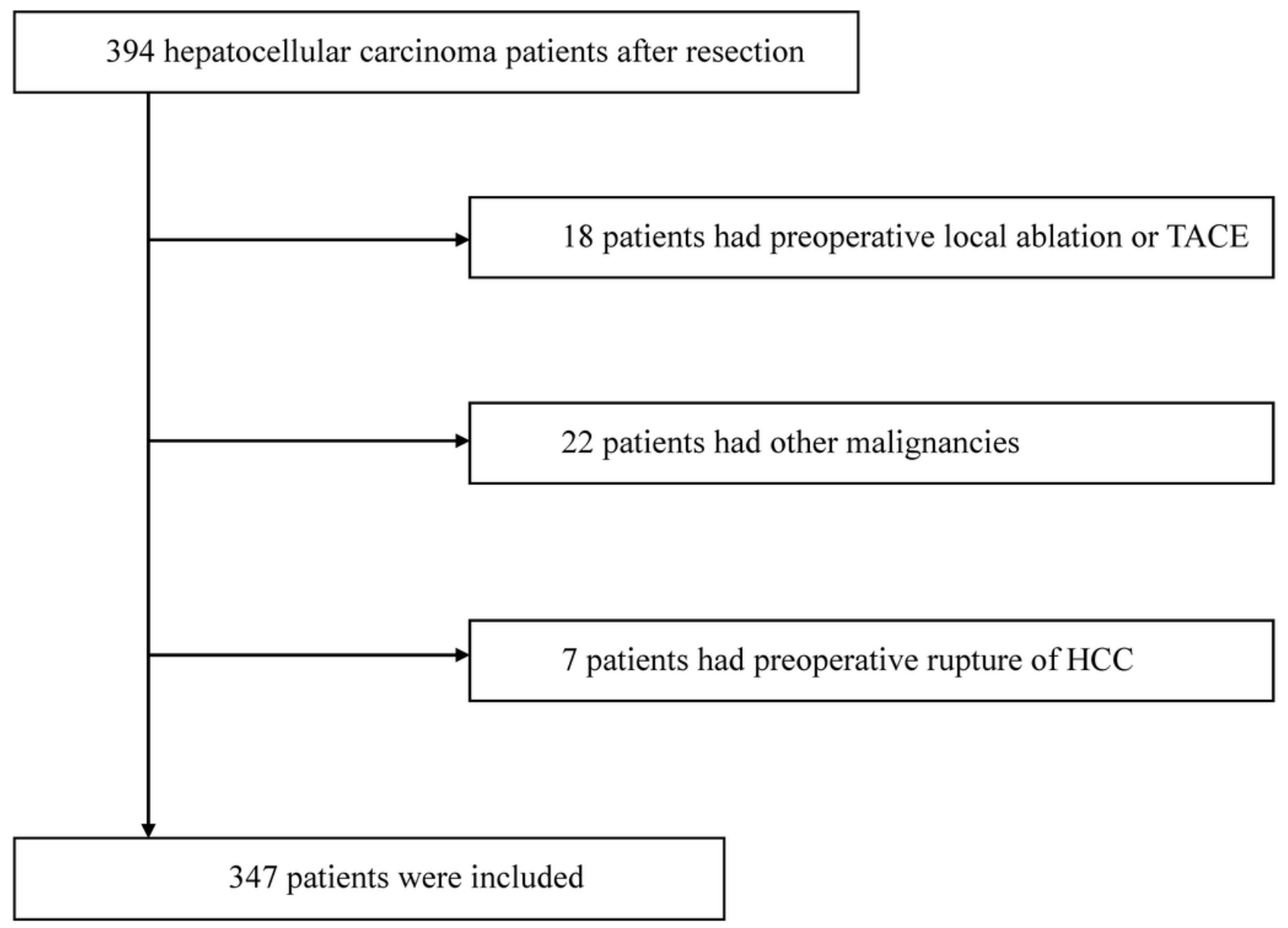

Figure 1

The flowchart of patient enrollment 


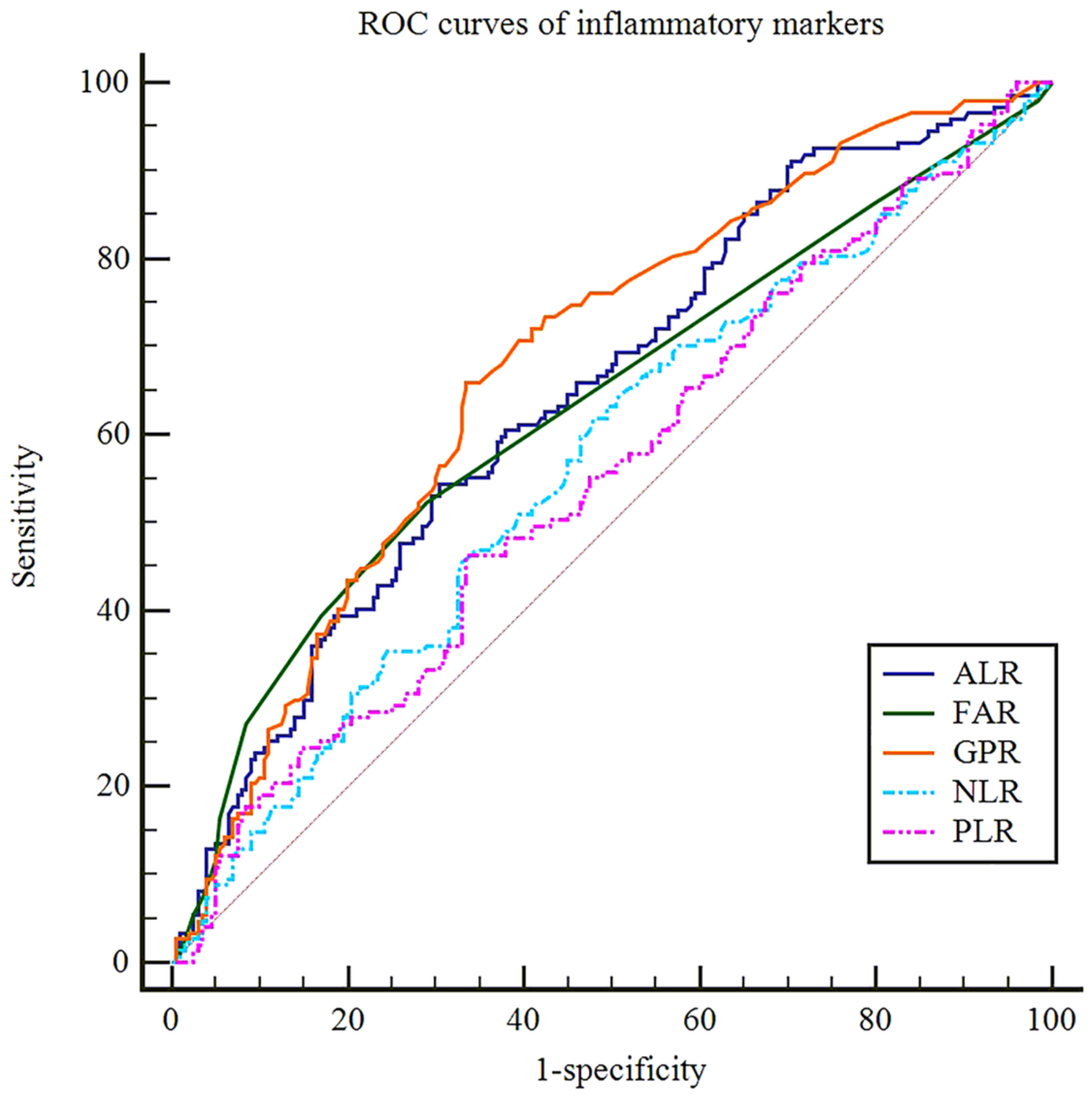

Figure 2

The ROC curves of NLR, PLR, GPR, ALR, and FAR 
a

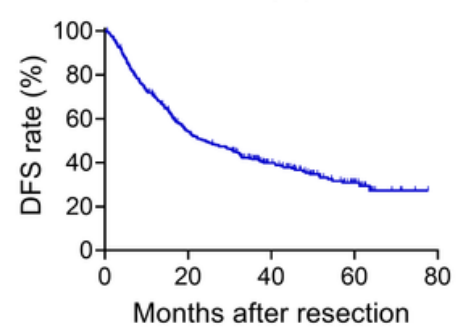

d

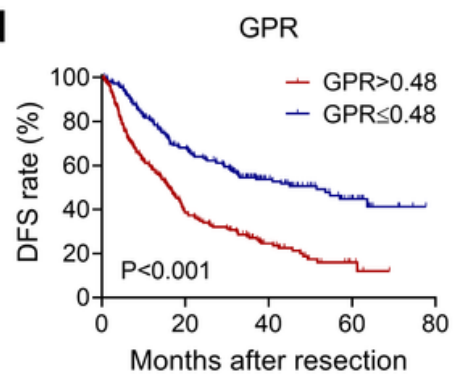

g

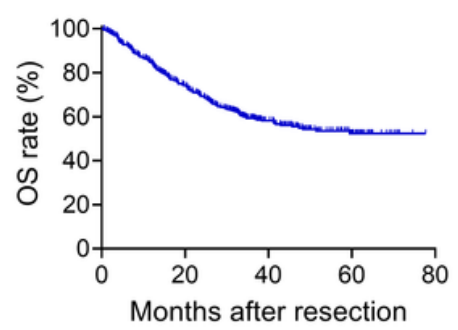

j

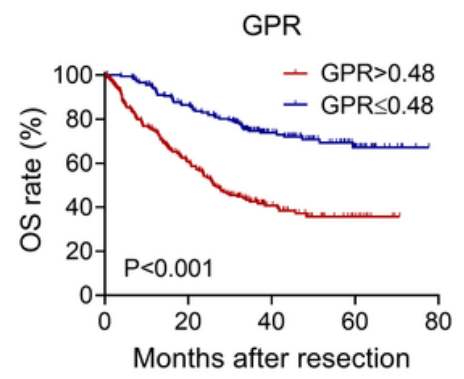

b

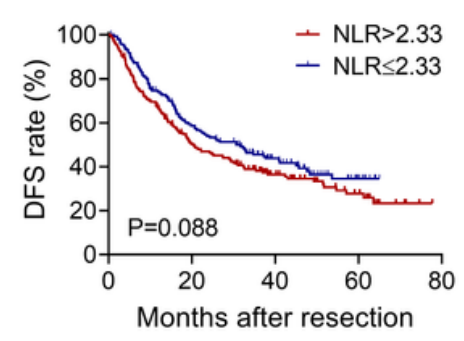

e

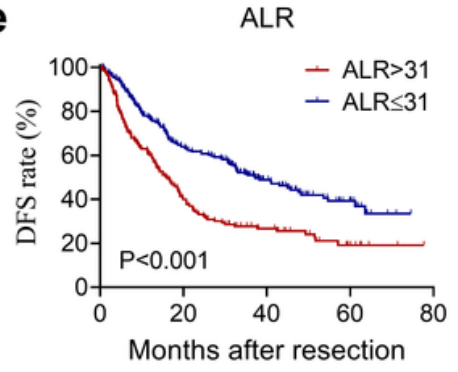

h

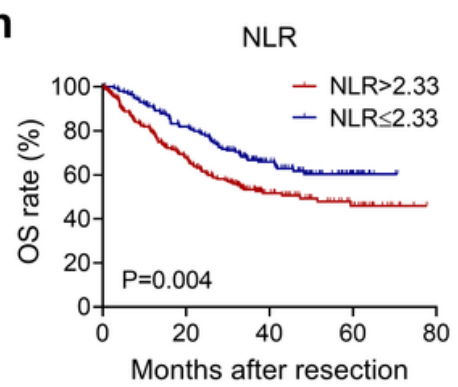

k

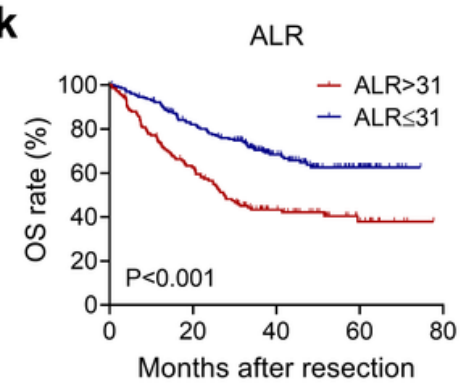

C

PLR

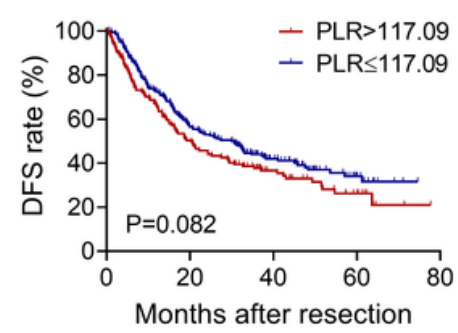

f

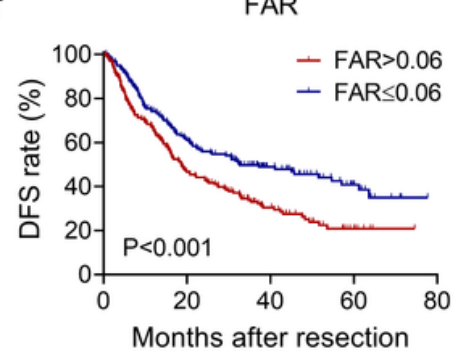

i

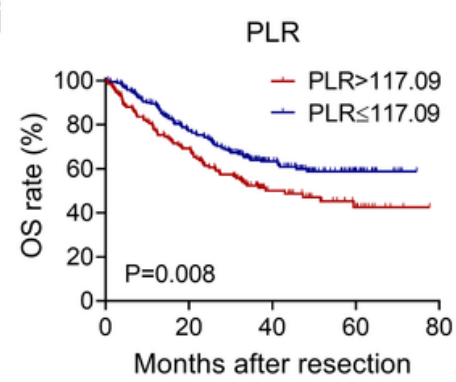

I

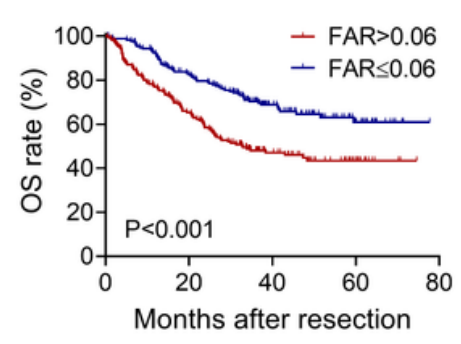

\section{Figure 3}

Kaplan-Meier analysis of (a) the total population, (b) NLR, (c) PLR, (d) GPR, (e) ALR, and (f) FAR for DFS区 Kaplan-Meier analysis of (g) the total population, (h) NLR, (i) PLR, (j) GPR, (k) ALR and (I) FAR for OS 
a

A-G-P score model in total population

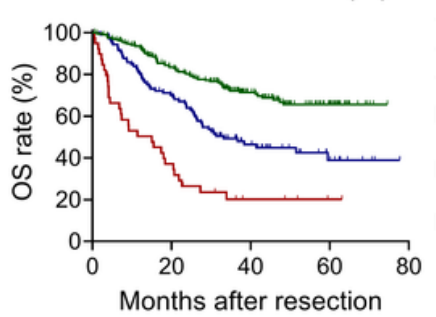

d

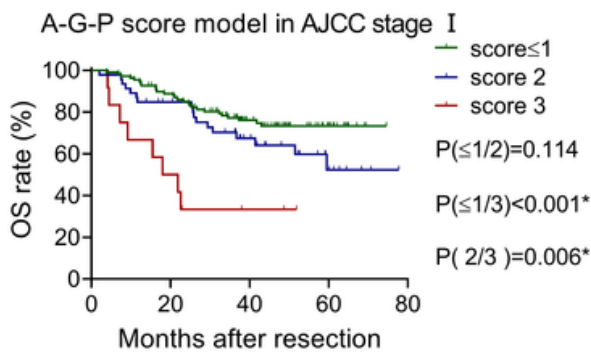

- score 3

$P(\leq 1 / 2)<0.001^{\star}$

$P(\leq 1 / 3)<0.001^{\star}$

$P(2 / 3)<0.001^{*}$ b

A-G-P score model in BCLC stage A

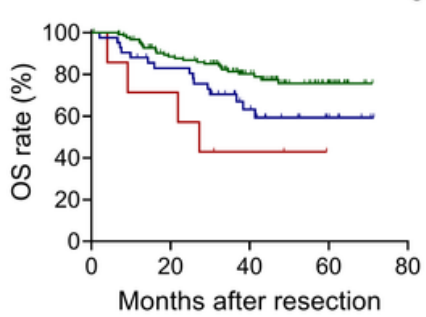

- score $\leq 1$

- score 2

- score 3

e

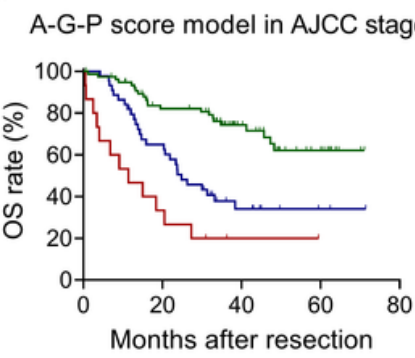

- scores 1

- score 2

- score 3
C

A-G-P score model in BCLC stage C

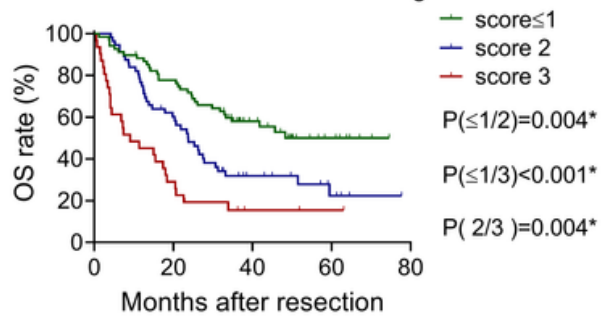

f

$P(\leq 1 / 2)<0.001^{\star}$

$P(\leq 1 / 3)<0.001^{*}$

$P(2 / 3)=0.029$

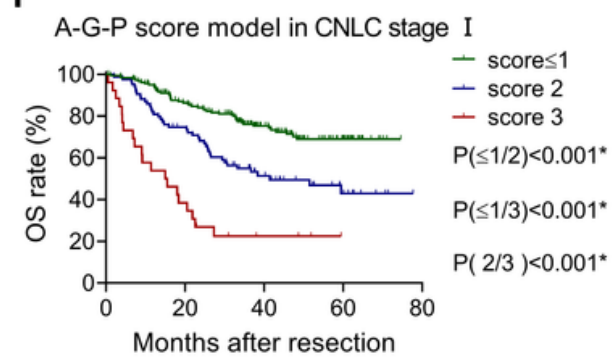

\section{Figure 4}

Kaplan-Meier analysis with log-rank test of A-G-P score model in (a) the total population (b) BCLC stage A (c) BCLC stage C (d) AJCC stage $\otimes(e)$ AJCC stage $\otimes$ (f) CNLC stage $\otimes$ for OS *statistical difference

a

GPR score model in total population

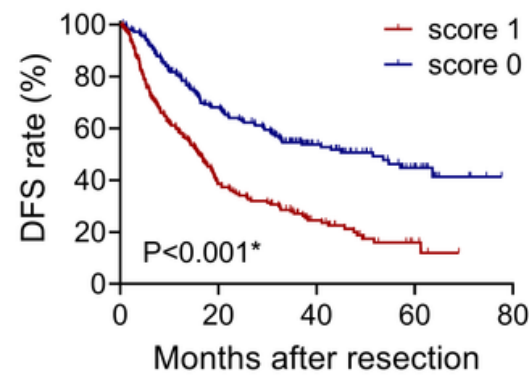

d

GPR score model in AJCC stage III

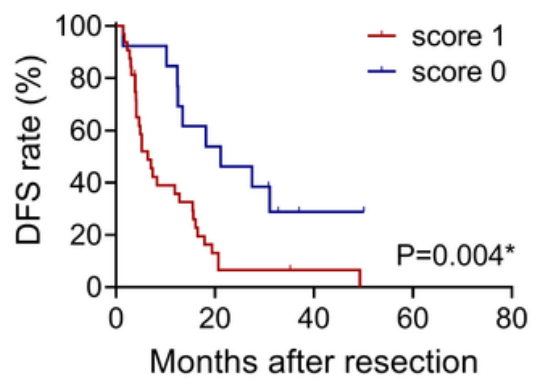

b GPR score model in BCLC stage C

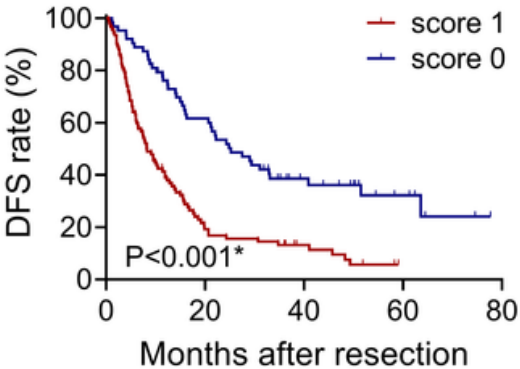

e GPR score model in CNLC stage I

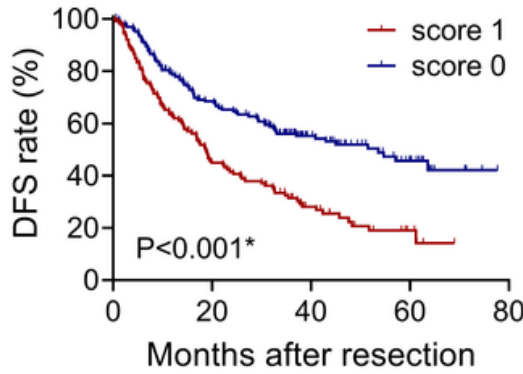

C GPR score model in AJCC stage II

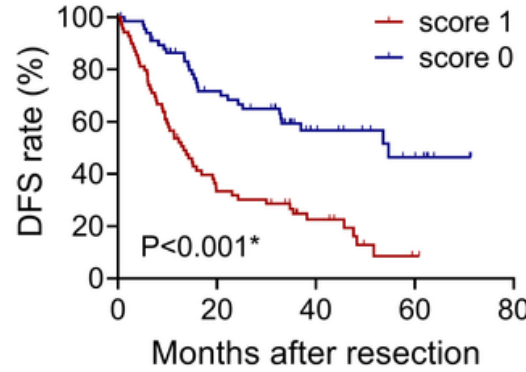

f GPR score model in CNLC stage III

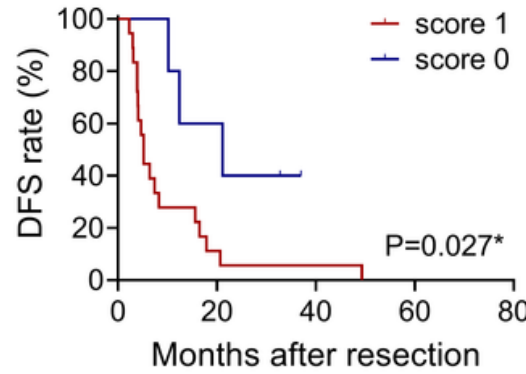

\section{Figure 5}


Kaplan-Meier analysis with log-rank test of GPR score model in (a) the total population (b) BCLC stage C (c) AJCC stage $\otimes$ (d) AJCC stage $\otimes$ (e) CNLC stage $\otimes$ (f) CNLC stage $\otimes$ for DFS *statistical difference

\section{Supplementary Files}

This is a list of supplementary files associated with this preprint. Click to download.

- AdditionalFiles.docx 